\title{
Combined treatment of vitamin $K$ and teriparatide on bone metabolism and biomechanics in rats with osteoporosis
}

\author{
YOUQING HUANG \\ Department of Pain Management, The Second Affiliated Hospital \\ of Kunming Medical University, Kunming, Yunnan 650101, P.R. China
}

Received July 6, 2017; Accepted October 24, 2017

DOI: $10.3892 /$ etm.2017.5420

\begin{abstract}
Postmenopausal osteoporosis is a degenerative disease caused by lack of estrogen whereby bone degeneration exceeds bone formation, resulting in loss of bone mass. Various drugs have been utilized in an attempt to ameliorate bone strength in such patients. The aim of the present study was to compare the effects of vitamin $\mathrm{K}$ or teriparatide alone and combined on bone metabolism and biomechanics in rats with osteoporosis. The ovaries of rats were excised to construct a rat model of osteoporosis. Rats were subjected to oral intake of vitamin $\mathrm{K}$ or subcutaneous injection of teriparatide or both for 8 weeks. ELISA was used to detect the content of carboxylated-type of osteocalcin (Gla-OC) and C-telopeptide of type I collagen (CTX-I) in serum. Bone density of shaft of femur and metaphyseal bone was measured. Three-point bending test was performed to analyze the load-deformation curve of femur. Undecalcified sections of femur were stained with toluidine blue to measure bone histomorphometric static, dynamic and bone resorption parameters. Compared with monotherapy, vitamin $\mathrm{K}$ combined with teriparatide significantly increased serum Gla-OC level and the number of osteoblast, decreased serum CTX-I level, reduced the number of osteoclasts and increased bone density and strength. This study showed that the efficacy of vitamin $\mathrm{K}$ combined with teriparatide is better than that of monotherapy. This combined treatment can promote bone formation, inhibit bone degradation, and improve bone density and strength.
\end{abstract}

\section{Introduction}

Postmenopausal osteoporosis is a degenerative disease caused by lack of estrogen. Bone degeneration in menopausal patients

Correspondence to: Dr Youqing Huang, Department of Pain Management, The Second Affiliated Hospital of Kunming Medical University, 374 Dianmian Road, Kunming, Yunnan 650101, P.R. China

E-mail: hyq06n@163.com

Key words: osteoporosis, vitamin K, teriparatide, bone metabolism, biomechanics exceeds bone formation, resulting in loss of bone mass. Osteoporosis can cause bone fragility and increase the risk of fracture. Multiple drugs have been proved to be able to improve bone strength in postmenopausal women. Those drugs include bisphosphonates, vitamin $\mathrm{D}$, vitamin $\mathrm{K}$ and teriparatide. The combination of these drugs can effectively improve the bone strength of some patients with osteoporosis (1).

Osteoblasts can synthesize non-collagenous bone protein, which is known as osteocalcin (OC). The $\gamma$-carboxylation of $\mathrm{OC}$ requires vitamin $\mathrm{K}$. Carboxylated-type of osteocalcin (Gla-OC) can effectively stimulate osteoblast to enhance bone mineralization (2). It has been reported that vitamin $\mathrm{K}$ can bind to steroid receptors to regulate gene transcription in osteoblast $(3,4)$. In addition, vitamin K not only enhances bone formation, but also inhibits bone degradation $(5,6)$. Vitamin $\mathrm{K}$ treatment can prevent bone loss and reduce the risk of fracture in women with postmenopausal osteoporosis $(7,8)$. Injection of teriparatide increases the number of osteoblasts, activates osteoblast activity, promotes osteoblast differentiation, increases trabecular bone volume and strength, and effectively stimulates bone formation $(9,10)$. Clinical trials have shown that teriparatide injection can increase bone density and reduce fracture risk in women with postmenopausal osteoporosis (11-14).

It has been reported that K-dependent $\gamma$-carboxylation of osteocalcin may enhance the efficacy of euproxine in bone repairing in rats with ovariectomy (15). Based on this, we hypothesized that vitamin $\mathrm{K}$ combined with teriparatide can promote bone formation and inhibit bone degradation, thereby improving bone metabolism. In this study, vitamin K combined with teriparatide was used to treat osteoporosis induced by ovariectomy in rats. The effects of the combination therapy on bone mineral density, bone strength and bone metabolic parameters were evaluated.

\section{Materials and methods}

Animal model. Fifty female Sprague Dawley rats (12 weeks old) were purchased from Charles River Laboratories (Beijing, Chia). The rats were separately raised in an SPF grade animal compartment with free access to water and food. Rats were randomly divided into the sham, ovariectomy (OVX), vitamin $\mathrm{K}(\mathrm{VK})$, teriparatine (TP), and vitamin $\mathrm{K}$ and teriparatide $(\mathrm{VK}+\mathrm{TP})$ group ( $\mathrm{n}=10$ rats per group). No significant difference in weight was found between the groups. Rats in 
A

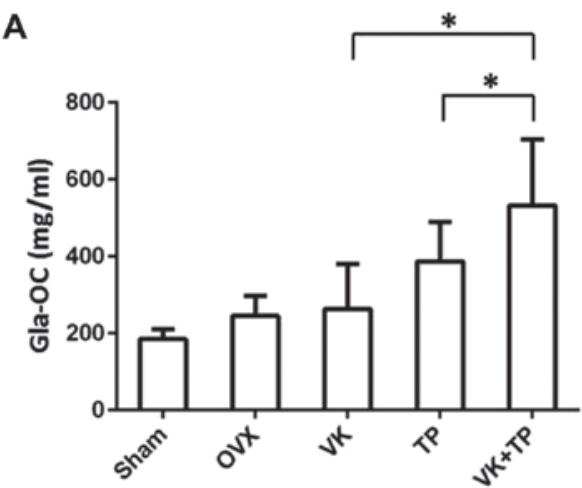

B

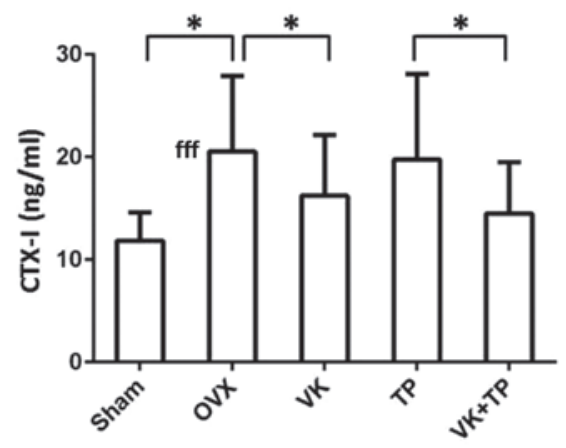

Figure 1. Bone metabolism indexes. (A) Serum carboxylated-type of osteocalcin (Gla-OC) levels (mg/ml) in each group of rats. (B) Serum C-telopeptide of type I collagen (CTX-I) levels $(\mathrm{ng} / \mathrm{ml})$ in each group of rats. ${ }^{*} \mathrm{P}<0.05$.

the OVX, VK, TP, and VK + TP groups were subjected to bilateral ovariectomy through the back. Surgical incision was also made in the same position of rats in the sham group but ovariectomy was not performed.

The study was approved by the Ethics Committee of the Second Affiliated Hospital of Kunming Medical University.

Drug administration. Rats in the VK and VK + TP groups were fed with vitamin K-deficient feed supplemented with vitamin $\mathrm{K}$ (30 mg/kg/day) and calcium (0.5/100 g feed). Rats in the other groups were fed with vitamin K-deficient feed supplemented with calcium $(0.5 / 100 \mathrm{~g}$ feed). Rats in the TP and VK + TP groups were injected subcutaneously with tropicotine hydrochloride (30 $\mu \mathrm{g} / \mathrm{kg}$ body weight), three times a week. Rats in the other groups were injected subcutaneously with an equal volume of normal saline. After drug treatment for 8 weeks, the rats were anesthetized by intraperitoneal injection of pentobarbital sodium $(50 \mathrm{mg} / \mathrm{kg})$ to collect blood $(10 \mathrm{ml})$ from femoral vein. Blood samples were centrifuged ( $800 \mathrm{x} \mathrm{g}$ for $20 \mathrm{~min}$ ) to separate serum and serum was stored at $4^{\circ} \mathrm{C}$. The right femur was frozen at $-80^{\circ} \mathrm{C}$ for bone density and biomechanical detection. The left femur was stored at $70 \%$ ethanol for bone histomorphometry.

Detection of bone metabolism parameters and bone mineral density. Venous blood was collected and enzyme-linked immunosorbent assay (ELISA) was used to detect Gla-OC (rat Gla-OC kit; Takara, Otsu, Japan) and C-telopeptide of type I collagen (CTX-I kit; RatLaps EIA, Boldon, UK) in serum. Rats were sacrificed by cervical dislocation, bilateral femur was collected from each rat, and soft tissue was removed. Dual-energy X-ray absorptiometry was used to scan the right femur to detect the bone density of the middle part of the femur and the position $1.5 \mathrm{~mm}$ from the growth plate of the femoral condyle.

Biomechanical testing. Lateral diameter of right femur was measured with a vernier caliper to calculate the area of the cross section of the midline of the femur. The three-point bending test was performed using a hydraulic servo fatigue testing machine (Servopulser; Shimadzu, Kyoto, Japan). The loading point is in the middle of the femur, with two fulcrums spaced $20 \mathrm{~mm}$ and a loading speed of $6 \mathrm{~mm} / \mathrm{sec}$.
The load-deformation curve was recorded, and elastic load, the maximum load and breaking load were calculated.

Bone tissue metrology. Bone tissue was fixed in $70 \%$ ethanol and embedded in glycol methacrylate to prepare undecalcified sections for toluidine blue staining. The tissues were observed and images were captured using a fluorescence microscope (DM LB2; Leica, Wetzlar, Germany). The measuring range was the secondary cancellous bone 1-4 mm below the growth plate of metaphysis of proximal femur. Images were randomly selected from the left, middle and right of the sections and six images were captured for each specimen. The morphological parameters of bone tissue were analyzed using pathological image analysis software. Static parameters included bone volume (BV), tissue volume (TV), and bone volume fraction (BV/TV). Dynamic parameters included bone formation rate (BFR) (bone turnover rate)/BV. Bone absorption parameters included number of osteoblast $(\mathrm{Ob})$, number of osteoblasts per unit area of bone trabecula, as well as surface area of osteoclast (Oc), number of osteoclasts per unit area of bone trabecula.

Statistical analysis. SPSS 19.0 software (Chicago, IL, USA) was used for statistical analysis. Data are expressed as mean \pm standard deviation. Comparisons of indexes between the groups were performed using the Student's t-test. $\mathrm{P}<0.05$ was considered statistically significant.

\section{Results}

Bone metabolism indexes. As shown in Fig. 1, the level of bone formation marker Gla-OC in OVX group was higher than that in the sham group. Gla-OC level in VK group was slightly higher than that in OVX group, but the difference was not statistically significant $(\mathrm{P}>0.05)$. Level of Gla-OC in TP group was significantly higher than that in OVX group $(\mathrm{P}<0.05)$. Level of Gla-OC in VK + TP group was significantly higher than that in VK and TP groups, individually $(\mathrm{P}<0.05)$. Level of bone degradation marker CTX-I was significantly higher in OVX group than in sham group $(\mathrm{P}<0.05)$. Level of CTX-I in VK group was significantly lower than that in OVX group $(\mathrm{P}<0.05)$. No significant difference in CTX-I level was found between the TP and OVX groups $(\mathrm{P}>0.05)$. It is noteworthy that CTX-I level in TP group was higher than that in VK group, 
A

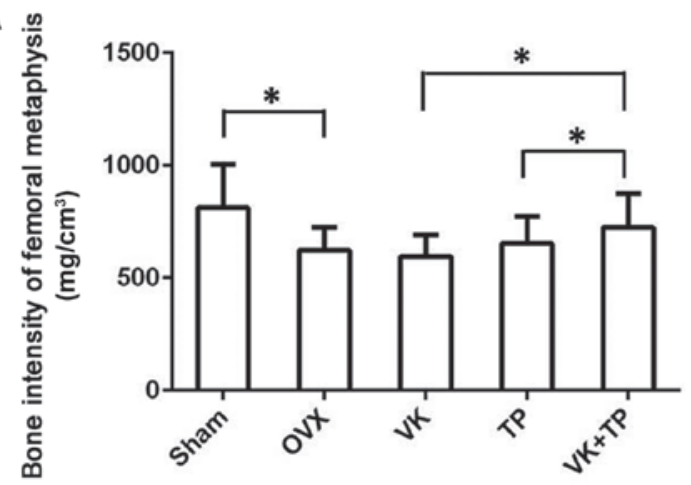

B

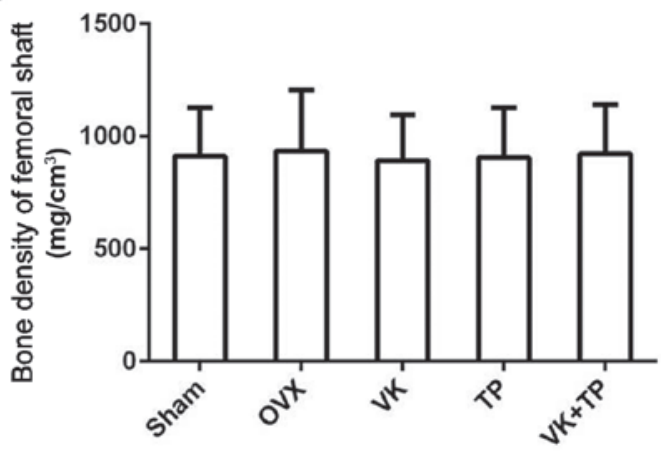

Figure 2. Bone intensity of femur. (A) Bone intensity of femoral metaphysis in each group $\left(\mathrm{mg}^{\mathrm{cm}} \mathrm{cm}^{3}\right)$. (B) Bone density of femoral shaft in each group $\left(\mathrm{mg} / \mathrm{cm}^{3}\right) .{ }^{*} \mathrm{P}<0.05$.

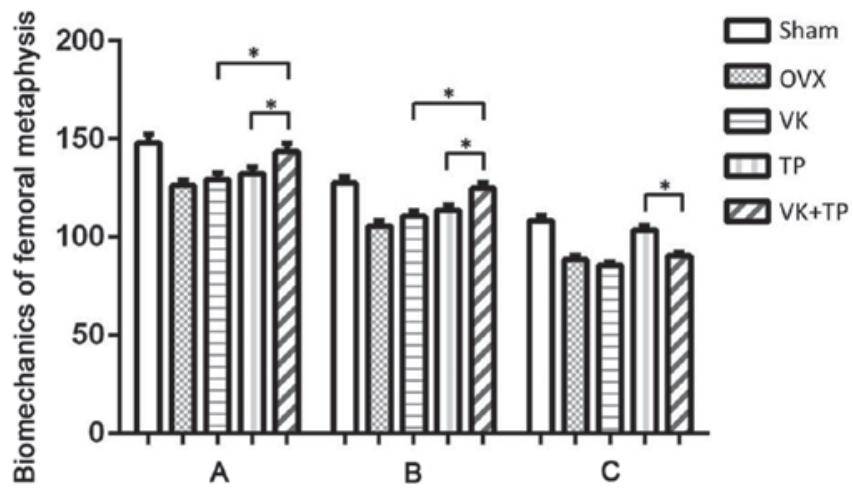

Figure 3. Biomechanics of femoral metaphysis. (A) Maximum load of femoral metaphysis $(\mathrm{N})$. (B) Breaking load of femoral metaphysis $(\mathrm{N} / \mathrm{mm})$. (C) Elastic load of femoral metaphysis (N). ${ }^{*} \mathrm{P}<0.05$.

but level of CTX-I in VK + TP group was significantly lower than that in TP group $(\mathrm{P}<0.05)$.

Bone density. As shown in Fig. 2, bone intensity of femoral metaphysis was lower in OVX group than that in the sham group $(\mathrm{P}<0.05)$, suggesting the occurrence of osteoporosis in ovariectomized rats. It is noteworthy that the bone mineral density of the VK group did not change significantly, and was slightly lower than that of the OVX group, the bone density of the TP group was higher than that of the OVX group. Bone mineral density of VK + TP group was significantly higher than that of the VK and TP groups $(\mathrm{P}<0.05)$. By contrast, there was no significant difference in bone density of the femoral shaft between groups $(\mathrm{P}>0.05)$.

Biomechanics. The elastic, maximum and breaking loads of femoral metaphysis in OVX group were lower than those in Sham group $(\mathrm{P}<0.05)$, suggesting severe osteoporosis in OVX group. The elastic and maximum loads of the VK and TP groups and the breaking loads of the TP groups were slightly higher than those of the OVX group, while those of VK + TP group were significantly higher than those of the OVX, VK and TP groups $(\mathrm{P}<0.05)$, suggesting the combined use of VK and TP recovered osteoporosis in ovariectomized rats (Fig. 3). Similar to the results of bone density of femoral shaft, no

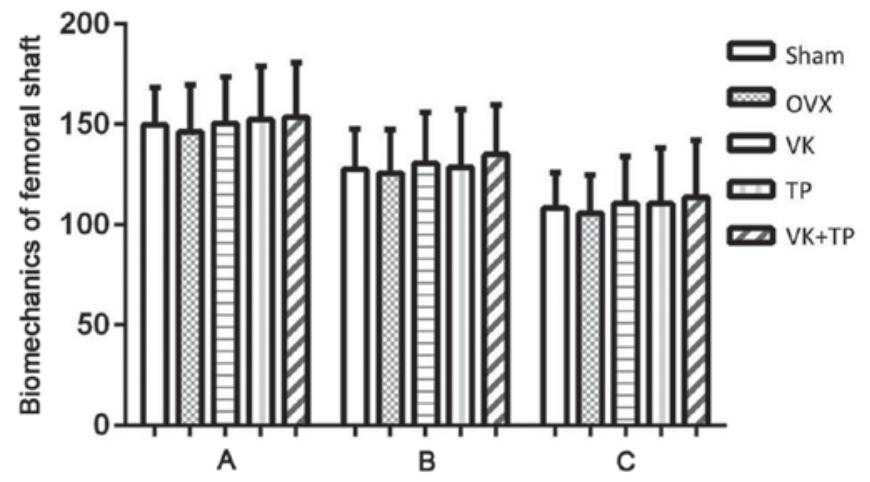

Figure 4. Biomechanics of femoral shaft. (A) Maximum load of femoral shaft $(\mathrm{N})$. (B) Breaking load of femoral shaft $(\mathrm{N} / \mathrm{mm})$. (C) Elastic load of femoral shaft $(\mathrm{N})$.

significant differences in the biomechanical parameters (elastic, maximum and fracture loads) of the femoral shaft were found between all groups (Fig. 4).

Bone histology. Changes in the static, dynamic, and bone resorption parameters are shown in Fig. 5. For the static parameters, bone volume fraction (BV/TV) in OVX group was significantly lower than that in sham group $(\mathrm{P}<0.01)$, suggesting that osteoporosis in ovariectomized rats. Compared to the OVX group, BV/TV of the TP group was significantly increased $(\mathrm{P}<0.05)$, but there was no significant change in VK group. By contrast, compared to OVX group, BV/TV was significantly increased in the VK + TP group $(\mathrm{P}<0.05)$, but no significant difference was found in BV/TV between the VK + TP and TP groups. For the dynamic parameters, the bone formation rate (BFR/BV) of OVX group was significantly higher than that of the sham, VK, TP and VK + TP groups $(\mathrm{P}<0.01)$. BFR/BV of the VK and TP groups was higher than that of sham group, and BFR/BV of VK + TP group was slightly higher than that of the sham group. Concerning bone absorption parameters, $\mathrm{Ob}$ was significantly higher in OVX group than in the sham group $(\mathrm{P}<0.05)$. Ob of the $\mathrm{VK}$ and $\mathrm{TP}$ groups was higher than that of the OVX group, but the difference was not statistically significant $(\mathrm{P}>0.05)$. Ob of $\mathrm{VK}+\mathrm{TP}$ group was significantly higher than that of OVX group $(\mathrm{P}<0.05)$. Oc of OVX group 

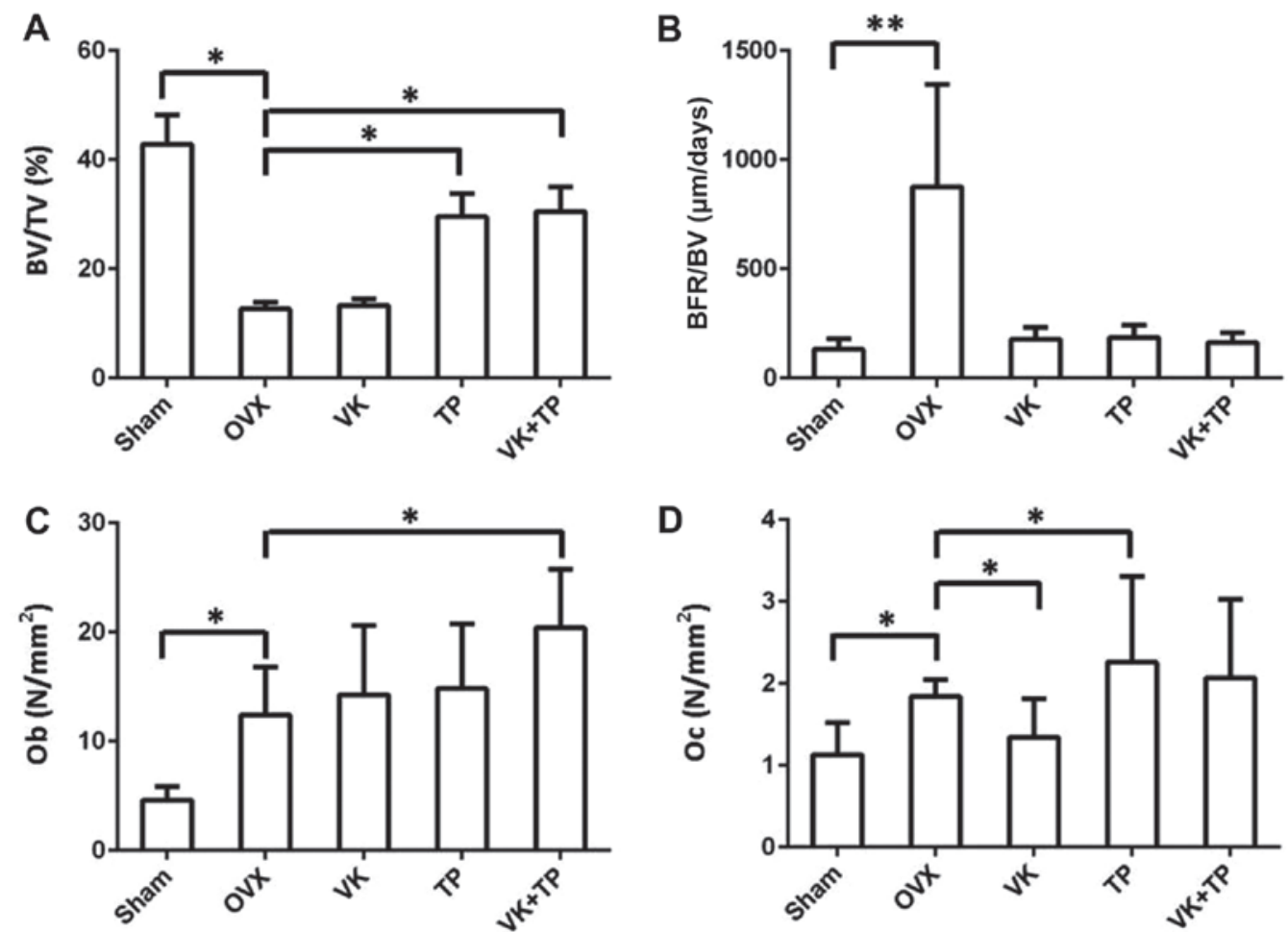

Figure 5. Bone histology parameters. (A) Bone volume (BV)/tissue volume (TV) (\%). (B) Bone formation rate (BFR)/BV ( $\mu \mathrm{m} / \mathrm{day})$. (C) Osteoblast (Ob) (N/mm²). (D) Osteoclast $(\mathrm{Oc})\left(\mathrm{N} / \mathrm{mm}^{2}\right)$. ${ }^{*} \mathrm{P}<0.05$ and ${ }^{* *} \mathrm{P}<0.01$.

was significantly higher than that of the sham group $(\mathrm{P}<0.05)$. Oc of VK group was significantly lower than that of the OVX group, and the Oc of the TP group was significantly higher than that of OVX group, while Oc of the VK + TP group was not significantly different from that of the OVX group $(\mathrm{P}<0.05)$.

\section{Discussion}

In this study, ovariectomy was performed to construct a rat model of osteoporosis. Effects of vitamin $\mathrm{K}$ and teriparatide monotherapy and combination therapy on bone metabolic parameters, bone mineral density, biomechanics and bone histomorphology were compared. Compared with monotherapy, vitamin K combined with teriparatide significantly increased the level of bone formation marker Gla-OC in serum and the number of osteoblasts. In addition, vitamin $\mathrm{K}$ combined with teriparatide increased the bone intensity of femoral metaphyseal and improved femoral tissue metrological parameters BV/TV and BFR. Results showed that the efficacy of vitamin K combined with teriparatide in the treatment of rats with osteoporosis is better than that of monotherapy. The combination treatment can promote bone formation by increasing the number of osteoblasts.

Rats that underwent ovariectomy were usually used as an animal model of osteoporosis. The model has good reproducibility, high bone turnover rate, accelerated bone formation and degradation, which can mimic postmenopausal osteoporosis in women caused by the lack of estrogen (16). In this study, ovariectomy resulted in the loss of femoral metaphyseal minerals and decreased mechanical strength, but did not affect the bone density and mechanical strength of the femoral shaft. This is consistent with previous studies whereby ovarian resection led to loss of cancellous bone (metaphyseal) without affecting the cortical bone (backbone) (17). In addition, the present study also found that ovariectomy increased the serum levels of Gla-OC and CTX-I, indicating accelerated bone turnover in rats with osteoporosis.

Following treatment with vitamin $\mathrm{K}$, levels of Gla-OC were increased, CTX-I levels were decreased, the number of osteoblasts was increased, while the number of osteoclasts was decreased in rats with osteoporosis, indicating that vitamin $\mathrm{K}$ promotes bone formation and inhibits bone degradation, which is consistent with previous studies $(5,6,18,19)$. Teriparatide can increase the number of osteoblasts in bone trabecula and activate cell activity in rats with osteoporosis, thereby promoting bone formation and increasing cancellous bone mass $(20,21)$. The findings of this study showed that teriparatide was able to promote bone formation by increasing serum levels of Gla-OC and number of osteoblasts. The mechanism of the effects of teriparatide on bone degradation remains unclear. Administration of teriparatide in ovariectomized rats did not alter the level of CTX-I, but increased the number of osteoclasts.

Compared with vitamin $\mathrm{K}$ or teriparatide monotherapy, vitamin $\mathrm{K}$ combined with teriparatide significantly increased the serum levels of Gla-OC and the number of osteoblasts, indicating that vitamin $\mathrm{K}$ combined with teriparatide can promote bone formation. Following treatment with vitamin $\mathrm{K}$, the serum CTX-I level was reduced and the number of osteoclasts was also reduced. Compared with trepiparone monotherapy, vitamin K combined with teriparatide reduced the serum level of CTX-I and the number of osteoclasts. These results suggest 
that both vitamin $\mathrm{K}$ monotherapy and in combination with teriparatone can inhibit bone degradation.

Additionally, the administration of teriparatide in rats with osteoporosis significantly increased bone density, maximum load and breaking load, which is consistent with previous studies $(22,23)$. By contrast, vitamin $\mathrm{K}$ can enhance fracture load, but cannot increase bone density (24). Vitamin K mainly enhances bone strength by increasing Gla-OC levels and therefore does not affect bone density (25). Compared to vitamin $\mathrm{K}$ monotherapy, vitamin $\mathrm{K}$ combined with teriparatide can significantly improve the bone density of femoral metaphysis. Compared with teriparatide monotherapy, vitamin $\mathrm{K}$ combined with teriparatide did not significantly improve bone density. However, compared with vitamin $\mathrm{K}$ or teriparatide monotherapy, vitamin K combined with teriparatide significantly increased the maximum and breaking loads. These results suggest that vitamin $\mathrm{K}$ combined with teriparatide can improve bone density and strength. Vitamin K monotherapy can reduce the femoral elastic load. Similarly, compared with teriparatide monotherapy, vitamin $\mathrm{K}$ combined with teriparatide also reduces the femoral elastic load. This suggests that vitamin $\mathrm{K}$ can enhance the flexibility of cancellous bone. Therefore, vitamin $\mathrm{K}$ combined with teriparatide, not only enhances bone strength, but also improves bone mass.

In conclusion, vitamin $\mathrm{K}$ combined with teriparatide can promote bone formation, and improve bone density and strength. Vitamin K combined with teriparatide combined can effectively prevent fractures in women with postmenopausal osteoporosis. However, future studies are required to elucidate the molecular mechanism of the effects of vitamin K combined with teriparatide on bone metabolism.

\section{References}

1. Orimo H, Nakamura T, Fukunaga M, Ohta H, Hosoi T, Uemura Y, Kuroda T, Miyakawa N, Ohashi Y and Shiraki M; A-TOP (Adequate Treatment of Osteoporosis) research group: Effects of alendronate plus alfacalcidol in osteoporosis patients with a high risk of fracture: The Japanese Osteoporosis Intervention Trial (JOINT) - 02. Curr Med Res Opin 27: 1273-1284, 2011.

2. Kobayashi M, Hara K and Akiyama Y: Effects of vitamin K2 (menatetrenone) and alendronate on bone mineral density and bone strength in rats fed a low-magnesium diet. Bone 35: $1136-1143,2004$

3. Tabb MM, Sun A, Zhou C, Grün F, Errandi J, Romero K, Pham H, Inoue $\mathrm{S}$, Mallick $\mathrm{S}$, Lin $\mathrm{M}$, et al: Vitamin $\mathrm{K} 2$ regulation of bone homeostasis is mediated by the steroid and xenobiotic receptor SXR. J Biol Chem 278: 43919-43927, 2003.

4. Igarashi M, Yogiashi Y, Mihara M, Takada I, Kitagawa H and Kato S: Vitamin K induces osteoblast differentiation through pregnane $X$ receptor-mediated transcriptional control of the Msx2 gene. Mol Cell Biol 27: 7947-7954, 2007.

5. Akiyama Y, Hara K, Kobayashi M, Tomiuga T and Nakamura T: Inhibitory effect of vitamin K2 (menatetrenone) on bone resorption in ovariectomized rats: A histomorphometric and dual energy X-ray absorptiometric study. Jpn J Pharmacol 80: 67-74, 1999.

6. Hara K, Akiyama Y, Nakamura T, Murota S and Morita I: The inhibitory effect of vitamin K2 (menatetrenone) on bone resorption may be related to its side chain. Bone 16: 179-184, 1995.

7. Inoue T, Fujita T, Kishimoto H, Makino T, Nakamura T, Nakamura T, Sato T and Yamazaki K: Randomized controlled study on the prevention of osteoporotic fractures (OF study): A phase IV clinical study of $15-\mathrm{mg}$ menatetrenone capsules. J Bone Miner Metab 27: 66-75, 2009.
8. Flore R, Ponziani FR, Di Rienzo TA, Zocco MA, Flex A, Gerardino L, Lupascu A, Santoro L, Santoliquido A, Di Stasio E, et al: Something more to say about calcium homeostasis: The role of vitamin K2 in vascular calcification and osteoporosis. Eur Rev Med Pharmacol Sci 17: 2433-2440, 2013.

9. Zhang K, Zhang FJ, Zhao WJ, Xing GS, Bai X and Wang Y: Effects of parathyroid hormone-related protein on osteogenic and adipogenic differentiation of human mesenchymal stem cells. Eur Rev Med Pharmacol Sci 18: 1610-1617, 2014.

10. Rosen CJ: The role of parathyroid hormone in the management of osteoporosis. Horm Res 64 (Suppl 2): 81-85, 2005.

11. Neer RM, Arnaud CD, Zanchetta JR, Prince R, Gaich GA, Reginster JY,Hodsman AB, Eriksen EF, Ish-Shalom S, Genant HK, et al: Effect of parathyroid hormone (1-34) on fractures and bone mineral density in postmenopausal women with osteoporosis. N Engl J Med 344: 1434-1441, 2001.

12. Miyauchi A, Matsumoto T, Sugimoto T, Tsujimoto M, Warner MR and Nakamura T: Effects of teriparatide on bone mineral density and bone turnover markers in Japanese subjects with osteoporosis at high risk of fracture in a 24-month clinical study: 12-month, randomized, placebo-controlled, double-blind and 12-month open-label phases. Bone 47: 493-502, 2010.

13. Boonen S, Marin F, Mellstrom D, Xie L, Desaiah D, Krege JH and Rosen CJ: Safety and efficacy of teriparatide in elderly women with established osteoporosis: Bone anabolic therapy from a geriatric perspective. J Am Geriatr Soc 54: 782-789, 2006.

14. Miyauchi A, Matsumoto T, Shigeta H, Tsujimoto M, Thiebaud D and Nakamura T: Effect of teriparatide on bone mineral density and biochemical markers in Japanese women with postmenopausal osteoporosis: A 6-month dose-response study. J Bone Miner Metab 26: 624-634, 2008.

15. Shimizu T, Takahata M, Kameda Y, Hamano H, Ito T, Kimura-Suda H, Todoh M, Tadano S and Iwasaki N: Vitamin Kdependent carboxylation of osteocalcin affects the efficacy of teriparatide (PTH(1-34)) for skeletal repair. Bone 64: 95-101, 2014.

16. Turner RT, Van der Steenhoven JJ and Bell NH: The effects of ovariectomy and 17 beta-estradiol on cortical bone histomorphometry in growing rats. J Bone Miner Res 2: 115-122, 1987.

17. Kalu DN: The ovariectomized rat model of postmenopausal bone loss. Bone Miner 15: 175-191, 1991.

18. Koshihara Y and Hoshi K: Vitamin K2 enhances osteocalcin accumulation in the extracellular matrix of human osteoblast in vitro. J Bone Miner Res 12: 431 438, 1997.

19. Iwasaki Y, Yamato H, Murayama H, Takahashi T, Ezawa I, Kurokawa $\mathrm{K}$ and Fukagawa M: Menatetrenone prevents osteoblast dysfunction in unilateral sciatic neurectomized rats. Jpn J Pharmacol 90: 88-93, 2002.

20. Hori M, Uzawa T, Morita K, Noda T, Takahashi H and Inoue J: Effect of human parathyroid hormone (PTH(1-34)) on experimental osteopenia of rats induced by ovariectomy. Bone Miner 3: 193-199, 1988.

21. Fox J, Miller MA, Newman MK, Metcalfe AF, Turner $\mathrm{CH}$, Recker RR and Smith SY: Daily treatment of aged ovariectomized rats with human parathyroid hormone (1-84) for 12 months reverses bone loss and enhances trabecular and cortical bone strength. Calcif Tissue Int 79: 262-272, 2006.

22. Iwaniec UT, Mosekilde L, Mitova-Caneva NG, Thomsen JS and Wronski TJ: Sequential treatment with basic fibroblast growth factor and PTH is more efficacious than treatment with PTH alone for increasing vertebral bone mass and strength in osteopenic ovariectomized rats. Endocrinology 143: 2515-2526, 2002.

23. Alexander JM, Bab I, Fish S, Müller R, Uchiyama T, Gronowicz G, Nahounou M, Zhao Q, White DW, Chorev M, et al: Human parathyroid hormone 1-34 reverses bone loss in ovariectomized mice. J Bone Miner Res 16: 1665-1673, 2001.

24. Matsumoto T, Miyakawa T and Yamamoto D: Effects of vitamin $\mathrm{K}$ on the morphometric and material properties of bone in the tibiae of growing rats. Metabolism 61: 407-414, 2012.

25. Iwamoto J, Sato Y, Takeda T and Matsumoto H: Bone quality and vitamin $\mathrm{K} 2$ in type 2 diabetes: Review of preclinical and clinical studies. Nutr Rev 69: 162-167, 2011.

This work is licensed under a Creative Commons Attribution-NonCommercial-NoDerivatives 4.0 International (CC BY-NC-ND 4.0) License. 